\title{
Editorial
}

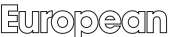

\section{New: The European Addiction Research Award}

European Addiction Research (EAR) and the European Federation of Addiction Societies (EUFAS) are jointly presenting the EUROPEAN Addiction Research Award. The award recognizes scientific excellence in clinical research in the addictions field and is endowed with 2500 Euros. One task linked to the award is to write a paper for EAR, which you will find in this volume of EAR.

In 2016, it was granted for the first time, and from now on, it will be given on a yearly basis. The recipient has to be a well-established European researcher who extensively contributed to the understanding and treatment of addictive disorders and their related harms.

EUFAS (www.eufas.net) is a federation currently consisting of 34 national and European Addiction Research Societies and Sections. Its mission is to harmonize the efforts of its members in preventing and treating addictive behaviors, to stimulate addiction research, and to suggest policies based on scientific evidence. To that end, EUFAS uses EAR as their preferred journal to submit surveys for publication, and provides its members in 25 European countries with information on progress in the field [2]. EUFAS is involved in establishing guidelines for diagnosis, treatment, and the dissemination of evidence-based approaches. To this end, EUFAS collaborates with national and European addiction research conferences, most recently with "Lisbon Addictions 2017" organized by European Monitoring Center for Drugs and Drug Addiction (EMCDDA). Here, EUFAS was involved in the EU-granted TWIST project to bring 147 young researchers and clinicians from 39 countries to the Lisbon confer- ence. The next meeting is scheduled for March 2018 at the annual conference of the European Psychiatry Association (EPA; www.europsy.net) in NICE in a joint event with the EPA section on Addictive Behaviors. Furthermore, EUFAS collaborates with the public sector on a European level and in countries of its members. In 2016, EUFAS teamed up with European Addiction Research (EAR) as its official journal. Whenever possible, EUFAS articles are presented in the public domain so that individuals of member societies and sections have easy access.

It is for us, as Editors-in-Chief of EAR and president of EUFAS, a great pleasure and honor to inform you that Prof. Dr. Karl Mann from the Central Institute of Mental Health, Mannheim, Germany, was the first recipient of the European Addiction Research Award 2016 and that Jürgen Rehm from the Institute for Mental Health Policy Research, CAMH, Toronto, Canada, and the Epidemiological Research Unit of the Technical University of Dresden, Germany, is the recipient of 2017. Their scientific status and global impact perfectly reflects the aim of this new European Addiction Research Award.

Karl Mann, MD, has published more than 500 peer-reviewed papers and authored or edited 20 books. They cover a broad range of scientific topics, and many have a focus on improving treatment modalities (for details see: Mann [4]). He has coordinated or participated in controlled trials covering alcoholism and smoking cessation. The fact that most of these activities took place in Europe is noteworthy because the vast majority of studies in our field come from the US. In line with his interest in improving treatment, he 
was also involved in several psychotherapy trials and coauthored a manual on alcohol-specific psychotherapy. These activities were bundled in Prof. Mann taking the lead in evidence-based treatment guidelines for alcohol use disorders (Mann etal. [5]) and tobacco dependencein German speaking countries (Batra et al. [1]).

Another longstanding interest of Karl Mann has been the structure and functioning of the human brain with alcoholism as a heuristic model. He detected structural changes in the brain of patients and provided evidence that alcoholic brain atrophy is partially reversible. He extended this work to controlled longitudinal studies looking at neuropsychological changes which go along with structural changes. Recently, he has used the same methodological approaches to study gambling and gaming behaviors (Dieter et al. [3]; Mann et al. [6]). Based on this work, he was invited to join the WHO working group for addiction diagnoses in ICD-11. Some of his current work is devoted to the attempt to optimize treatment response (Precision Medicine) for homogeneous subgroups of patients (Mann et al. [8]; Mann et al. [7]). Linked with the award, Karl Mann submitted a manuscript to EAR published in the current volume (Mann et al. [9]).

Jürgen Rehm, PhD, has been author or co-author of more than 900 peer-reviewed articles and more than 20 monographs. His work has been recognized by inclusion into Thomson Reuters highly cited researchers for the last years (top $1 \%$ of researchers for most cited documents in their specific field globally in the 10 years prior). The scientific work of Dr. Rehm has focused on substance use epidemiology and population health research, with contributions to the Global Burden of Disease and Injury studies (GBD 2016 Risk Factor Collaborators, 2017; Rehm et al. [14]) and the WHO Global Status Reports on Alcohol and Health (WHO, 2014); and on effectiveness and cost-effectiveness evaluations of treatment and other interventions, both from the perspective of the client (Rehm and Barbosa [11]) and the society (Rehm et al. [11]). His work includes models to judge clinical significance over medium term based on the usual short time trials with follow-ups of 1 year of less (e.g., Roerecke et al. [12]); these models have led to the approval of new pharmaceuticals by the European Medicines Agency [13-15].

With this first award, both EUFAS and EAR intend to start a long-standing tradition, honoring key European addiction researchers, and giving visibility to the importance of addiction research in Europe. Our common mission is to contribute to the sharing of research and clinical excellence, and connecting addiction professionals throughout Europe. We invite you all to be in contact and will inform you of future activities via our websites and publications in European Addiction Research.

Prof. Geert Dom, MD, President of EUFAS

Prof. Wim van den Brink, MD, Editor in chief EAR

Prof. Falk Kiefer, MD, Editor in chief EAR

\section{References}

1 Batra A, et al: S3 guideline: screening, diagnostics, and treatment of harmful and addictive tobacco use. Sucht 2016;62:139-152.

2 Bramness J, Mann K, Wurst F: Marketing status and perceived efficacy of drugs for supporting abstinence and reducing alcohol intake in alcohol use disorders: a survey among European Federation of Addiction Societies in Europe. Eur Addict Res 2016;22:318-322.

-3 Dieter J, et al: The role of emotional inhibitory control in specific internet addiction - an fMRI study. Behav Brain Res 2017;324:1-14.

4 Mann K: Addiction research centres and the nurturing of creativity. Department of addictive behaviour and addiction medicine, central institute of mental health, Mannheim, University of Heidelberg. Addiction 2010; 105:2057-2061.

5 Mann K, Batra A, Fauth-Bühler M, Hoch E; Group G: German guidelines on screening, diagnosis and treatment of alcohol use disorders. Eur Addict Res 2017;23:45-60.

6 Mann K, et al: Comorbidity, family history and personality traits in treatment seeking pathological gamblers compared with healthy controls. Eur Psychiatry 2017;42: $120-128$

7 Mann K, et al: Precision medicine in alcohol dependence: a controlled trial testing pharmacotherapy response among reward and relief drinking phenotypes. Neuropsychopharmacology 2017, Epub ahead of print.

-8 Mann K, et al: Predicting naltrexone response in alcohol-dependent patients: the contribution of functional magnetic resonance imaging. Alcohol Clin Exp Res 2014;38:27542762.

-9 Mann K, Aubin H-J, Witkiewitz K: Reduced Drinking in Alcohol Dependence Treatment, What Is the Evidence? Eur Addic Res 2017;23: 219-230.

10 Rehm J, Barbosa C: The cost-effectiveness of therapies to treat alcohol use disorders. Exp Rev Pharmacoecon Outcomes Res 2017, Epub ahead of print.

11 Rehm J, Shield KD, Rehm MX, Gmel G, Frick $\mathrm{U}$ : Modelling the impact of alcohol dependence on mortality burden and the effect of available treatment interventions in the European Union. Eur Neuropsychopharmacol 2013;23:89-97.

12 Roerecke M, Sørensen P, Laramée P, Rehm J: Clinical relevance of nalmefene versus placebo in alcohol treatment: reduction in mortality risk. J Psychopharmacol 2015;29:11521158.

13 GBD 2016 Risk Factors Collaborators: Global, regional, and national comparative risk assessment of 84 behavioural, environmental and occupational, and metabolic risks or clusters of risks, 1990-2016: a systematic analysis for the Global Burden of Disease Study 2016. Lancet 2017;390:1345-1422.

14 Rehm J, Mathers C, Popova S, Thavorncharoensap $M$, Teerawattananon $\mathrm{Y}$, Patra J: Global burden of disease and injury and economic cost attributable to alcohol use and alcohol use disorders. Lancet 2009;373:22232233.

15 World Health Organization: Global Status Report on Alcohol and Health. Geneva, World Health Organization, 2014. 